Somnologie $2019 \cdot 23: 58$

https://doi.org/10.1007/s11818-019-0190-z

Online publiziert: 30. Januar 2019

(c) Springer Medizin Verlag GmbH, ein Teil von Springer Nature 2019

\title{
Danke an alle Gutachter 2018
}

Die Redaktion dankt allen Experten und Expertinnen für Ihre Unterstützung im Jahr 2018 als wissenschaftliche Gutachter für die Somnologie. Durch ihren Einsatz garantieren sie im Rahmen des Peer-Review-Verfahrens der eingereichten Manuskripte die Qualität der Zeitschrift.

Michael Arzt

Tatjana Crönlein

Christina Dirks

Torsten Eggert

Thomas Erler

Rene Fischer

Wolfgang Galetke

Annika Gieselmann

Robert Göder

Svenja Happe

Holger Hein

Clemens Heiser

Simon-Dominik Herkenrath

Michael Herzog

Nikolaus Netzer

Ekkehart Paditz
Thomas Penzel

Roland Popp

Winfried Randerath

Kneginja Richter

Angelika Schlarb

Barbara Schneider

Christoph Schoebel

Richard Schulz

Ulrich Sommer

Kai Spiegelhalder

Jens Spiesshoefer

Boris A. Stuck

Michael Urschitz

Veronika Vielsmeier

Sebastian Zaremba 